\title{
Is safety of childhood growth hormone therapy related to dose? Data from a large observational study
}

\author{
Lars Sävendahl', Effie Pournara², Birgitte Tønnes Pedersen ${ }^{3}$ and \\ Oliver Blankenstein ${ }^{4}$
}

1Department of Women's and Children's Health, Karolinska Institutet and Pediatric Endocrinology Unit, Karolinska University Hospital, Stockholm, Sweden, ${ }^{2}$ Global Medical Affairs, Novo Nordisk Health Care AG, Zurich, Switzerland, ${ }^{3}$ Epidemiology, Novo Nordisk A/S, Søborg, Denmark, and ${ }^{4}$ Institute for Experimental Pediatric Endocrinology, Charité-University Medicine Berlin, Berlin, Germany

Correspondence should be addressed to L Sävendahl Email

lars.savendahl@ki.se

Following publication of this article in the May issue of the journal (vol 174, Issue 5; pp 681-691) the authors wish to clarify the following point:

This article reports the occurrence of all serious adverse events (SAEs) in the low-risk group and serious adverse drug reactions (SADRs) in the intermediate- and high- risk groups due to cerebrovascular events during growth hormone (GH) treatment in childhood. Therefore, as described in the 'Statistical analysis' section of the publication, we evaluated all events reported as SAEs or SADRs (as appropriate for the respective risk group) under the MedDRA SOC classification system as nervous system or cardiovascular events.

The focus of the publication is on the safety of GH in the low-risk group. As documented in the publication, we found no cerebrovascular events reported as SAEs in the low-risk group during our study. Additionally, we found no cerebrovascular events reported as SADRs in the intermediate- and high-risk groups. However, in one high-risk group patient, two cerebrovascular events (brain stem infarction and cerebral infarct) reported as SAEs were identified. This patient was diagnosed with craniopharyngioma at 12 years of age and started hormone replacement therapy, including GH treatment for hypopituitarism, 6 months after the initial diagnosis. The patient experienced a brain stem infarction at 14 years of age followed by a cerebral infarct at 17 years of age. The two events were assessed by the treating physician as not related to GH therapy; therefore, these two events were not reported in the manuscript. The authors apologise for the omission.

๑) 2017 European Society of Endocrinology Printed in Great Britain
Published by Bioscientifica Ltd. 